\title{
BioSense 2.0 Governance: Surveying Users and Stakeholders for Continued Development
}

\author{
Joe Gibson ${ }^{1}$, Bryant Thomas Karras² and Gideon S. Gordon*3 \\ ${ }^{1}$ Marion County Public Health Department, Indianapolis, IN, USA; ${ }^{2}$ Washington State Department of Health, Shoreline, WA, USA; \\ ${ }^{3}$ eHealth, Association of State and Territorial Health Officials, Arlington, VA, USA
}

\section{Introduction}

The CDC's BioSense 2.0 system is designed with a user-centered approach, where the needs and requests of the users are part of its continued development. User requirements were gathered extensively to help design BioSense 2.0 and users continue to submit feedback which is used to make improvements to the system. However, in order to ensure that these needs are gathered in a formal and ongoing way, the BioSense 2.0 Governance Group, comprised primarily of state and local public health representatives, was established to advise the CDC on the development of BioSense 2.0.

The Governance Group (GG) understands that to make recommendations having direct relevance and utility to the community, they must engage public health jurisdictions which use BioSense 2.0. To that end, the GG has conducted three surveys of the BioSense 2.0 community. The survey results will help inform the group's prioritized recommendations to the CDC. In this presentation we discuss the findings and lessons learned from these surveys.

\section{Methods}

Survey 1, conducted in December 2012, assessed preferences concerning software packages that might be placed in the BioSense 2.0 cloud-space to complement the existing system. The survey listed software options for syndromic surveillance, statistical analysis and geovisualization. Respondents rated their knowledge about and preferences for each software option. All users of BioSense were invited to take the survey. Responses were normalized by jurisdiction, and a final score was calculated for each option.

Survey 2, conducted in April 2013, assessed user preferences concerning many, detailed functionality options for BioSense 2.0. The items to be rated were derived from a comprehensive list of desired functionalities for syndromic surveillance systems, developed by NACCHO's BioSurveillance Workgroup.

Survey 3, conducted in 2013, assessed stakeholder prioritization of a broader range of development areas, comprehensive but not overly detailed. The GG discussed BioSense 2.0 development priorities in several meetings, generating 20 overarching topics. Respondents were asked to select their top 10 topics, then rank those in order of importance. Just one response per jurisdiction received the questionnaire. The rankings for each of the 20 topics will be averaged and weighted according to the number of jurisdictions choosing each option and the values given for them.

\section{Results}

Reports with result of the surveys can be found at the Governance webpage (http://tinyurl.com/BiosenseGovernance).

Survey 1: $33 \%$ of users responded from $3 / 4$ of all BioSense $2.0 \mathrm{ju}-$ risdictions. The number of respondents per jurisdiction varied widely. The most preferred software was ArcGIS (geo-visualization category), SAS (statistical analysis), and ESSENCE (syndromic surveillance analysis). Users found the survey to be fairly easy to complete.

Survey 2: $19 \%$ of users participated in this survey, with at least one response from $95 \%$ of BioSense 2.0 jurisdictions. In some categories, respondents showed significant preference for particular choices (e.g, data sources and coding/classifying) while in other categories all choices were nearly equally preferred. Users found the survey to be complex and time-consuming.

Survey 3: $92 \%$ of BioSense 2.0 jurisdictions responded to this survey. The top 5 priorities, in order of descending priority, were enhancing analytical tools, increased granular views, data quality awareness tools and meta-data visibility, data visualization, and increased data sources or types.

\section{Conclusions}

Understanding the needs of the public health community for BioSense 2.0 development is critical to the initiative's success. The GG is challenged to balance information detail against respondent burden. The results of Survey 1 were clear, but the scope only addressed one particular issue of many. Survey 2 was designed to be more comprehensive, but was viewed as too detailed and, in some cases, offered little clarity for prioritization.

As a result, the approach taken in survey 3 , while more comprehensive than the prior surveys, encapsulated issues in just 20 topics. Ranking choices against each other helps crystallize the relative importance of issues. Finally, asking for only one response per jurisdiction allowed a clear egalitarian approach.

The Governance Group's methods of gathering input from the BioSense 2.0 community will continue to change as Biosense 2.0 evolves. The Governance Group will continue to evaluate and refine its approaches to get a clear assessment of the needs and desires of the public health community for system development.

\section{Keywords}

BioSense; BioSense 2.0; syndromic surveillance; governance; platform

*Gideon S. Gordon

E-mail: sgordon@astho.org 